Dario Prola

Istituto di Comunicazione Specialistica e Interculturale, Facoltà di Linguistica Applicata, Università di Varsavia

\title{
"Dove abitano il tempo, il silenzio e la voce delle chiese”: la Roma di Maria Kuncewiczowa
}

\section{Nel filone novecentesco del viaggio in Italia}

Con la riconquista dell'indipendenza nel 1918 e la normalizzazione dei rapporti internazionali, viene meno la figura del pellegrino polacco ramingo per l'Europa. Se si assiste al nascere in Polonia del fenomeno del moderno "turismo" - certo ancora privilegio di pochi - il viaggio viene nuovamente concepito come un'avventura umanistica e formativa, artistica o intellettuale nata dalla volontà di conoscere e di conoscersi. I contributi dall'etnologia (con i lavori di Bronisław Malinowski in testa) offrono agli scrittori sguardi e prospettive culturali inedite, mentre la letteratura di viaggio si esprime in forme diverse che vanno dal romanzo al diario, dalla cronaca al moderno reportage. Si sviluppa nel periodo interbellico la cosiddetta "literatura faktu" (non-fiction, letteralmente "letteratura del fatto") dove gli scrittori avvertono la necessità di documentare la realtà in cui vivono o viaggiano, sia interna alla Polonia che esterna ${ }^{1}$. In questo senso i reportage di Maria Kuncewiczowa Dyliżans warszawski (La diligenza varsaviana, 1935) e Miasto Heroda

1 Sul reportage nel periodo interbellico si veda Kąkolewski 1992: 930- 935; Piechota, Stępnik 2004; Glensk 2014. 
(La città di Erode,1939), testimonianza di un viaggio in Palestina costituiscono due esempi emblematici di questa nuova sensibilità e dimensione del viaggiare. Come scrive Teresa Wilkoń [2006: 137], molti scrittori iniziano ad esprimere il loro interesse in forme inedite, più consone ai tempi e al nuovo modo di spostarsi; vengono messe da parte le forme lunghe e tradizionali come il poema o il memoriale di viaggio, che richiedono una maggiore elaborazione e documentazione, in favore di altre più brevi, come versi descrittivi, appunti diaristici, lettere, piccoli quadretti di genere.

In questo contesto di grande apertura, l'Italia continua a essere una delle mete privilegiate degli scrittori polacchi. Il mito arcadico dell'Italia, e quello di Roma in particolare [Biliński 1992; Barycz 1965], così importante per gli scrittori romantici e poi per quelli modernisti, per quanto elaborato con minore vivacità rispetto all'epoca precedente [Kozińska-Donderi 2003: 96] continua a svilupparsi nella letteratura delle nuove generazioni di scrittori, assumendo valenze ed espressioni conformi alla nuova epoca. Molti scrittori, come Jan Parandowski in Mój Rzym (La mia Roma, 1970)2 o Zdzisław Morawski in Rzym: portrety i szkice (Roma: schizzi e ritratti, 1921), restano fedeli alla tradizione iniziata da Winckelmann e compiono i propri viaggi di erudizione sulle tracce dell'antico e delle culture classiche. Se per loro l'Italia continua a rappresentare una seconda patria ideale, in linea con il sentimento espresso dalle parole di K.C. Norwid: "Polonus natus, civis Romanus", altri scelgono di mantenere un atteggiamento critico nei confronti del mito, ora attraverso un distanziamento (come fa Jarosław Iwaszkiewicz nei vivaci e ironici reportage dall'Italia che trovarono spazio sulle pagine di "Wiadomości Literackie" negli anni 1932-1933) ora spingendosi fino a una demitizzazione o dissacrazione (come quella messa in atto da Witold Gombrowicz nel suo Wjazd do krainy wtoskiej [Ingresso in terra italiana], scritto in seguito a un viaggio del 1938).

Questa differenziazione degli sguardi sull'Italia e sul viaggio in Italia prosegue anche dopo la Seconda guerra mondiale a par-

2 Si tratta di articoli e saggi scritti nel periodo interbellico e raccolti in volume solo nel 1970. 
tire dai diari di viaggio di Jerzy Stempowski apparsi su "Kultura" nel 1948, ai saggi postumi raccolti in Od Berdyczowa do Rzymu (Da Berdyczów a Roma, 1971) dello stesso Stempowski, alla raccolta di novelle e schizzi di Jan Parandowski Godzina śródziemnomorska (Ora mediterranea, 1949). Continua a fiorire il reportage letterario, le cui prime attestazioni nel periodo postbellico sono Passeggiata: szkice wtoskie (Passeggiata: schizzi italiani) di Jan Bielatowicz, pubblicato a Roma nel 1947, e Kroniki Assyżu (Cronache di Assisi, 1947) di Roman Brandstaetter. Rispetto a queste due opere, caratterizzate da un approccio più personale e intimo all'Italia e al suo patrimonio culturale, i reportage pubblicati in Polonia mostrano una chiara impostazione ideologica marxista; ne sono un esempio Spotkania wtoskie (Incontri italiani, 1949) di Marian Brandys, Pożegnanie $z$ Italia (Addio all'Italia, 1954) di Julian Stryjkowski, il volume di schizzi di Marcin Czerwiński Tradycje włoskie. Szkice literackie (Tradizioni italiane. Schizzi letterari, 1954).

Con il disgelo e la fine del realismo socialista, nelle pagine dei viaggiatori polacchi le osservazioni di matrice sociale tornano a concedere spazio al discorso culturale e il mito italico viene ripreso anche nella prospettiva di un dialogo con una tradizione culturale spesso avvertita come ingombrante. Una prima attestazione in questo senso è Ksiażka o Sycylii (Libro sulla Sicilia) di Jarosław Iwaszkiewicz. In quest'opera, pensata e scritta durante la guerra ma pubblicata solo nel 1956, l'autore rievoca i primi viaggi in Italia come una sorta di personale percorso iniziatico attraverso l'arte e il paesaggio del Belpaese. Sempre in questo filone "tradizionale" vanno segnalati anche Barbarzyńca w ogrodzie (Un barbaro nel giardino, 1962) di Zbigniew Herbert e Mitśródziemnomorski (Il mito mediterraneo, 1962) di Mieczysław Jastrun, dove lo sguardo all'Italia e al mito classico si intreccia con una riflessione sulla condizione della civiltà occidentale che, segnata dalle grandi tragedie del $\mathrm{xx}$ secolo, sembra giunta al punto di non ritorno. In queste opere, gli scrittori si sforzano di trovare nel patrimonio artistico e culturale dell'Italia le radici comuni europee (discorso che implica sempre un'autoriflessione sulla identità polacca) e una chiave di lettura per interpretare il caos morale della contemporaneità. A tutto questo si somma altresì la ricerca di un approccio inedito e il tentativo di sin- 
tesi individuale dell'arte e della civiltà italiana, aspetti riscontrabili per esempio in opere come Listy do Pani Z. (Lettere alla Signora Z., 1957-1961) di Kazimierz Brandys e Podróże do Włoch (Viaggi in Italia, 1977) di Jarosław Iwaszkiewicz. È proprio in questo filone dove la riflessione degli scrittori si incunea tra individualità e universalità, passato e presente, che si colloca anche il libro "italiano" di Maria Kuncewiczowa.

\section{Notatki włoskie: genesi, genere e contenuti}

Il bisogno di raccontare la realtà senza infingimenti porta la Kuncewiczowa fin dagli anni Trenta a iniziare un filone saggistico che - intrecciandosi a quello romanzesco - si sviluppa per tutta la sua lunga carriera ${ }^{3}$. Si tratta di opere ibride, dove l'elemento autobiografico, il dato di costume, il fatto storico convivono con la rappresentazione di una realtà sulla quale l'autrice non teme di esprimere giudizi chiari e perentori, da persona non abituata a scendere a compromessi.

L'ultimo frutto di questa vasta e brillante produzione saggistica è un libro interamente dedicato all'Italia, paese dove - a partire dal 1970 e fino agli ultimi anni di vita - la scrittrice si recava con il marito per periodi di riposo più o meno lunghi, soprattutto in inverno e in primavera. La loro destinazione era prevalentemente Roma, anche se occasionalmente si spingevano in Meridione: Ischia, Amalfi, Taormina sono altri luoghi che hanno lasciato traccia in questo scarno volumetto di appunti e riflessioni di viaggio. Apparsi dapprima sparsamente su varie riviste ("Twórczość”, "Więź”, "Literatura”), vennero raccolti nel volume Notatki włoskie. Przezrocza

Questa produzione - aperta nel periodo prebellico con le opere già citate Dyliżans warszawski e Miasto Heroda - prosegue con: Klucze (Chiavi, 1943), racconto, tra diario e reportage, del pellegrinaggio attraverso l'Europa in guerra; Odkrycie Patusanu (La scoperta di Patusan, 1958), raccolta di schizzi, saggi e reportage apparsi in prevalenza su rivista nel periodo interbellico; Don Kichot i niańki (Don Quijote e le balie, 1965), appunti, schizzi e quadretti ispirati da un viaggio in Spagna. Verso la fine della vita la scrittrice proseguirà seguendo un'ispirazione più profondamente autobiografica con Fantomy (Spettri, 1971), Natura (1975) e il volume oggetto di questo studio. 
(Appunti italiani. Istantanee) dato alle stampe nel 1985 - quattro anni prima della morte dell'autrice - dalla casa editrice cattolica PAX e altre due volte dopo il 1989. Solamente l'edizione del 2010 è stata integrata dei brani soppressi dalla censura comunista (gli interventi di quest'ultima sono riportati nel testo e i frammenti espunti sono riprodotti in nota) ${ }^{4}$.

Il titolo contiene una chiara indicazione di genere, prova a definire una relazione tra la realtà e la sua rappresentazione. Nel rapporto tutto sensoriale che l'autrice instaura con il mondo, il primato è dello sguardo ("Przezrocza" significa 'diapositive', anche se, da un punto di vista estetico, la traduzione più adeguata potrebbe essere 'istantanee'). L'importanza che l'osservazione ricopriva nella sua arte è messa in rilievo dalla stessa Kuncewiczowa che dice in un'intervista: "Guardare è in effetti la mia natura, la mia passione, io vivo attraverso lo sguardo, attraverso il tocco" [Zaworska 1983: 46]. Tuttavia l'osservazione e l'interpretazione della realtà è solo uno dei piani del discorso letterario e ha un carattere piuttosto pretestuoso, finalizzato - proprio come in Fantomy e Natura - a dar spazio a tutta una serie di riflessioni metafisiche e religiose nonché all'affioramento della dimensione della memoria.

Da un punto di vista formale, non appare azzardato ricondurre questo libro alla silva rerum, genere ampiamente praticato già nell'antica letteratura polacca ${ }^{5}$ e ripreso - secondo nuove modalità - anche nella seconda metà del Novecento. La silva rerum, nella sua variante contemporanea, si caratterizza come un genere non pianificato, i cui testi sono di fatto privi di un ordine interno degli elementi che ne faciliti la fruizione. Si tratta di una tipologia formale dove la mancanza di norme estetiche fisse, l'assenza di un inquadramento in un preciso genere letterario, permettono all'au-

Sulla base di questa edizione è condotta la presente analisi. Le citazioni dal volume sono indicate con il solo numero di pagina a seguire. Qualora non diversamente specificato tutte le traduzioni dal polacco in questo saggio sono mie.

5 Nell'ambito dell'antica letteratura polacca la silva rerum ('il bosco delle cose') indicava una sorta di almanacchi che contenevano scritti da vario genere: annotazioni sulla vita della nobiltà polacca, testi letterari in prosa e in poesia, diari, relazioni di viaggio o di avvenimenti importanti, ma anche ricette e curiosità di vario genere. 
tore un'eccezionale apertura tematica e a ogni sorta di digressione e annotazione. Unitamente al suo carattere autobiografico, la caratteristica essenziale della silva è la frammentarietà e l'imprevedibilità. La narrazione si sviluppa dunque liberamente, suscitata da associazioni e reminiscenze, dalle infinite possibilità a cui si apre il testo ${ }^{6}$.

In Notatki wtoskie - opera ibrida che nasce dall'incontro tra il reportage letterario e il diario scritto a posteriori ${ }^{7}$ - il magma del materiale narrativo trova nella versione finale una ordinata organizzazione in capitoli costituiti di sottocapitoli densamente concisi - da una a tre pagine - incentrati su un episodio, un paesaggio, una persona e su tutto ciò che può essere spunto per le riflessioni dell'autrice. Lo stile, tra il lirico e il saggistico, è estremamente curato, testimonianza di un accurato lavoro di rielaborazione.

Il libro offre nel complesso un ritratto "a caldo" e del tutto personale del Belpaese sullo sfondo dei complessi anni Settanta e costituisce un' importante e ancora attuale riflessione sullo stato di crisi della civiltà occidentale contemporanea, sui passati splendori dell'Italia, sulla minaccia - oggi globale - dell'estremismo e del terrorismo. L'impulso che dà vita a questo libro è il bisogno, intellettuale ed esistenziale, di trovare un ordine nel caos del mondo contemporaneo.

Gli eventi intorno ai quali ruota la riflessione di Kuncewiczowa sono il rapimento di Aldo Moro e l'elezione di Karol Wojtyła al soglio pontificio. Due fatti di importanza capitale per il popolo italiano e per quello polacco, ma che vanno compresi in prospettiva globale in quanto conseguenza del lacerante conflitto tra i due blocchi che si contesero i destini del mondo per la maggior parte del xx secolo. Fatti che hanno molto in comune, anche per tutti i parallelismi suscitati dal loro accostamento. Leader carismatico, Karol Wojtyła - il primo papa a capire fino in fondo l'importanza della comunicazione e a ser-

6 La silva rerum, in particolare nella sua variante diaristica, negli ultimi anni della Polonia comunista assecondava l'esigenza di verità del lettore. La letteratura di finzione era infatti sospettata di alterare i tratti della realtà. Da parte sua lo scrittore, investito del ruolo di testimone esclusivo del mondo, facendo riferimento alla memoria individuale, al fatto vissuto, cercava di fornire al pubblico un frammento di verità, seppur personale e talvolta arbitraria [Czapliński 1998: 77].

7 Teresa Świętosławska [1997: 105] parla di "roman personnel nella sua più pura forma di journal intime romantico". 
virsi dei media con cognizione -, leader senza carisma, Aldo Moro, politico tutt'altro che capace di smuovere le masse, uomo forse più portato alla solitudine claustrale che all'agone politico. Due uomini politici uniti dal Cristianesimo: uno guida della cristianità ma capace di dialogare con il mondo laico, con le istituzioni dello stato italiano, con i rappresentanti di tutte le confessioni, l'altro - ex presidente del consiglio, segretario della DC - fervente cattolico che seppe venire a patti con il comunismo parlamentare in nome di un "compromesso storico" che non fu mai del tutto realizzato. Due leader vittime della lotta ingaggiata dal terrorismo contro le istituzioni: uno uscitone vincitore, capace di sfruttare abilmente l'attentato di cui fu vittima per rafforzare la propria immagine e il senso della missione apostolica di fronte alle masse, l'altro sconfitto poiché sacrificato sull'altare della "religione di Stato", eletto suo malgrado a martire dei poteri istituzionali da un'oligarchia politica che prima lo lasciò cadere e poi, ipocritamente, lo beatificò.

Giovanni Paolo II appare nell'opera come autentico contraltare allo stato, l'uomo che - secondo l'autrice - aveva portato alla rinascita del cattolicesimo e alla restituzione di un'autorità morale alla Chiesa cattolica. Il giudizio di Kuncewiczowa è appassionato e convinto, sostenuto da una fede autentica - mai dogmatica levigata dal dubbio e da una lunga ricerca. Giudizio non privo di orgoglio patriottico - l'elezione di Wojtyła è definita "miracolo sul Tevere" - e sostenuto da autentica e candida speranza: "Questo Papa porta la croce del messianesimo polacco e forse riuscirà a portare al mondo la fede nella resurrezione" (138).

Il rapimento di Moro è raccontato a caldo, dal vivo (la scrittrice si trovava a Roma nella tragica primavera del 1978). Kuncewiczowa, che si serviva correttamente della lingua italiana, ci riporta a quei tragici giorni commentando le allarmanti notizie dei giornali, i drammatici comunicati che i brigatisti facevano pervenire a media e istituzioni. Dai notiziari televisivi le giunge quel mondo "sporco del sangue di passanti uccisi, della saliva dei demagoghi

8 Riferimento all'espressione Cud nad Wista ("Miracolo sulla Vistola") con la quale viene ricordata la vittoriosa battaglia di Varsavia, inizio della controffensiva che portò alla fine della Guerra sovietico-polacca (1919-1921), al favorevole trattato di Riga e al successivo armistizio. 
e della polvere della democrazia" (69). Secondo la scrittrice l'“affaire Moro" - per citare il titolo del celebre saggio che Leonardo Sciascia scrisse nell'estate di quell'anno - mette a nudo uno dei più grossi pericoli cui è approdata la civiltà occidentale: dimostra 1' importanza dello Stato e la non importanza dell'uomo" (126). Un giudizio impietoso e lapidario, che spazza via in un colpo solo qualsiasi lettura dicotomica del mondo, ogni facile contrapposizione o alternativa tra sistemi politici e sociali, tra "buoni" e "cattivi", noi e loro, stato e terrorismo. " [...] non è forse lo stato un'organizzazione terroristica che desidera la morte dei suoi avversari? Quale cittadino e quale paese sopravviverà al terrore dello stato se non riconoscerà quale suo supremo tribunale quella forza che alcuni chiamano Dio, altri civiltà, altri ancora coscienza?" (215) si domanda Kuncewiczowa. Più che un politico, Aldo Moro è per lei soprattutto un uomo, con tutte le debolezze, le paure, i desideri, il biologico istinto vitale, vittima paradossale di un sistema che lui stesso ha contributo a creare.

\section{L'immagine di Roma}

Lo sfondo sul quale si disputa questa drammatica lotta Stato ed Antistato (secondo la narrazione ufficiale) o tra Stato e Uomo (secondo la scrittrice) è una Roma dall'atmosfera surreale dove - mentre i bambini continuano a giocare nei parchi e gli adulti a frequentare bar e mercati - da qualche parte, in una prigione domestica, si consuma una tragedia dalla portata immediatamente storica e universale. La capitale in cui affondano le radici della civiltà cristiana ed europea non è una città rassicurante: vi si combatte una terribile "guerra civile". "Ma di quale casa [dom] si tratta? - si chiede l'autrice nel saggio "Wojna domowa” - Dell'Italia? Dell'Europa? Del mondo?" (106). Di chi è guerra che incendia la nostra casa? Perché se Roma è la casa di tutti, allora lo è anche la guerra che vi si combatte.

Nel saggio "Być u siebie" (Essere a casa) un truce fatto di cronaca, per nulla straordinario in Italia - un africano senzatetto bruciato vivo nel suo giaciglio di fortuna tra una chiesa e un immondezzaio - la spinge a riflettere sul significato e sui rapporti tra essere ed abitare: 
$[\ldots]$ anche a me capita di essere una senzatetto e potrei essere bruciata in qualsiasi momento da quelli che disprezzano chi dorme sull'immondezzaio altrui. Nel ventennio interbellico lo scrittore [... Juliusz Kaden-Bandrowski accomiatandosi dal romanticismo poté dichiarare trionfalmente che la Polonia aveva finalmente il suo immondezzaio. Su questo immondezzaio di concetti, tradizioni e necessità Jerzy ha costruito la nostra casa a Kazimierz, casa che fummo costretti a lasciare nel settembre del 1939, quando le sue pareti erano appena state innalzate. [...] Finalmente nel 1958 sono ritornata [...] Che strano! Durante tutti gli anni postbellici trascorsi sulla Vistola, in quella "nostra" casa, nuovamente abitata, riscaldata, amata, non mi sono sentita neppure per un solo giorno a casa, così come mi sono sentita negli appartamenti in affitto di prima della guerra. Ora avverto sempre un qualche intruso alle mie spalle. E questa impressione la divido con gli autoctoni a Roma, in Inghilterra, in America, ovunque mi spinga il destino. È dunque finita l'era dei propri immondezzai? Sbagliano i burloni a bruciarli insieme alla loro fauna straniera: nessuno infatti può più dirsi a casa sua (158-159).

Si tocca qui un altro, urgente problema che rende questo libro così attuale: il problema della casa, non solo nel senso di locum, di spazio esistenziale dove programmare e condurre la propria vita, ma soprattutto inteso come spazio storico, culturale e identitario. Per la Kuncewiczowa l'estraneità è condizione e problema cruciale dell'uomo contemporaneo, e questa sua riflessione - già alla base del fortunato romanzo che la rese famosa: La straniera $(1935)^{9}$ - venne da lei raffinata nei lunghi anni delle peregrinazioni postbelliche ${ }^{10}$.

9 Tradotto in ben 16 lingue, la versione italiana di Cudzoziemka uscì nel 1939: La straniera, Milano, Mondadori (Traduzione di Renato Poggioli). Il libro ristampato varie volte fino all'ultima edizione (Bompiani 1984) - fu preceduto dalla traduzione del romanzo del 1928 Twarz mężczyzny: Il volto dell'uomo, RomaLecce, G. Cafaro, 1935 (Traduzione di Silvana Lupo). Non esistono altre traduzioni italiane di opere della Kuncewiczowa eccetto il racconto Próba innej formy: ja i on: Prova di un'altra forma: Io-lui pubblicato in Statuti 1988: 154-157. 
Roma è la città contemporanea per antonomasia, locus orribilis dove - nel marasma e nell'impazzare della violenza politica - si consuma la crisi della civiltà dell'uomo occidentale. Se da un lato è la " [...] fortezza della cristianità circondata dalle brigate rosse e da quelle nere, città dove il numero dei suicidi supera quello delle vocazioni" (177), dall'altro è solo una delle tante violente e caotiche capitali occidentali. È proprio su questo apparente paradosso che si gioca l'ambivalente immagine della Roma di Kuncewiczowa: in questa città dove tutti sono stranieri e chiunque si sente - in un certo senso - a casa, si consuma una tragedia particolare (perché tutta italiana) e universale (perché la possibilità di venire stritolato dallo stato o di venire bruciati con le proprie cose potrebbe toccare a chiunque e ovunque). Eppure la fragilità dell'uomo e dei prodotti del suo lavoro, fisico ed intellettuale, contrasta con la natura di questa città, continuamente sospesa tra eternità e decadenza. Ma ritrovare l'eternità di questa città - frantumata in una miriade di scorci, gesti, parole - è ancora possibile?

Sì, perché nel suo panorama i fori, i templi, gli acquedotti, i circhi si fondono in modo fluido, simultaneo, con le cattedrali della cristianità, con il trascorrere proprio ed altrui. No, perché la realtà materiale e metafisica scorre accanto ai circhi e alle chiese in modo così violento che il suo chiasso sovrasta le preghiere che, sul Tevere come sulla Vistola, sono rivolte all'eterno (197).

Questo frammento indica chiaramente come la Roma di Maria Kuncewiczowa sia una città bifronte sospesa tra sacro e profano, trascendenza e immanenza; è Gerusalemme e Babilonia al tempo stesso. Si vedano i toni apocalittici della seguente descrizione che

schiavitù polacca, all'età di cinque anni si traferì a Varsavia. All'inizio del secondo conflitto mondiale lasciò la Polonia iniziando un lungo pellegrinaggio che si protrarrà per molti anni; abitò in Francia, Inghilterra, Stati Uniti, dove insegnò letteratura polacca all'Università di Chicago. Negli anni Settanta, dopo averne trascorsi ventitré all'estero, ritornò a Kazimierz sulla Vistola, dove la sua casa ospita attualmente un museo dedicato alla sua memoria e a quella del marito, lo scrittore e studioso Jerzy Kuncewicz. 
sembra richiamarsi al più genuino antiurbanismo della tradizione polacca. Proprio mentre la scrittrice passa sotto la colonna Traiana monumento in onore dell'imperatore che aveva esteso alla Dacia i confini dell' impero - in piazza del Gesù "il cadavere del dignitario democristiano aspetta il tocco della mano dell'amata Noretta”.

Soffiava un vento impregnato di benzina, s'addensavano i lamenti e i fischi delle automobili pigiate sulla strada dal Colosseo a via del Corso, la follia delle macchine e dei macchinisti era cresciuta a un punto oltre il quale non c'è che la morte improvvisa e inattesa. E proprio allora scese sulla città un inimmaginabile secondo di silenzio. Qualcosa come il volo di un'enorme falena o il respiro di una luna bianca. E proprio allora sentii per la seconda volta in questa città di cimiteri il piccolo trotto dell'asinello sotto il peso del Messia viaggiatore. "Dove conduci, o Signore? - chiedo. Ma ritorna il frastuono e in esso naufraga la risposta divina (136).

In questa città dove lo scorrere dell'esistenza quotidiana si fa Storia, dove convergono le contraddizioni e antinomie del nostro tempo, è più forte il richiamo dell'eternità. Pur essendo "città di cimiteri”, Roma resiste al trascorrere del tempo e delle generazioni, a quella fragilità umana che la Kuncewiczowa - prossima alla fine della sua lunga vita - misurava su se stessa. Le riflessioni sulla vecchiaia, sul precipitare della vita nella morte, minuto dopo minuto, si susseguono su queste pagine: "Quanto poco mi interessava l'eternità fintanto che il sangue scorreva a ritmo indisturbato nelle mie vene, le gambe non si intorpidivano di pomeriggio, la mia testa emetteva pensieri ben associati, la memoria restituiva in un lampo parole e fatti dimenticati!" (197). Non lo spazio dunque è la categoria dominante in quest'opera ma il tempo: il tempo psichico, il tempo storico, l'eternitàn ${ }^{11}$ Incontriamo in diverse occasioni, tra

Come scrive Teresa Świętosławska [1997: 104]: “Gli Appunti italiani registrano ciò che è possibile: le proprie impressioni fissate nella memoria. Il tempo è la misura della durata dei fenomeni e per questo una sua unità può essere la 'diapositiva': fisica, biologica, trascendente; oggettiva e soggettivata; interna ed esterna; psichica (vissuta) e ontologica (resa cosciente)". 
queste pagine, Maria Kuncewiczowa perduta nella contemplazione del panorama della città dall'alto, spesso sul morire del giorno, alla ricerca di quel varco che le permetta di intravedere, racchiuso come in un'istantanea, il senso della sua eternità.

Come un tempo sono andata sul Gianicolo a guardare Roma al tramonto. Come un tempo sono stata abbagliata da quel fenomeno separato dalle sue radici, sospeso nel tempo come un arazzo indistruttibile intessuto di scene e figure sbiadite, sporco da vicino, magnifico da lontano. Ma ora provavo a indovinare in quale punto della mappa si trovava la stanza delle torture del presidente della Democrazia Cristiana (117).

Così come il frastuono di Babilonia è interrotto per un istante dal richiamo del sacrum, allo stesso modo la ricerca dell'eternità, di cui il panorama di Roma dall'alto è segno terreno e materiale, è disturbata dal pensiero che laggiù, in un imprecisato punto della topografia urbana, si consuma uno dei drammi del nostro tempo.

Tuttavia Roma non è solo la città dove si cristallizzano i miti di Aldo Moro e Karol Wojtyła, dove va in scena la Storia: la scrittrice è anche interessata agli abitanti della Città eterna, alla loro esistenza quotidiana, sempre la stessa, nonostante il fluire del tempo. Kuncewiczowa è testimone di una Roma vista dal basso, dalla prospettiva della strada, dei mercati, della folla; è una flâneur che guarda, osserva, si interroga sull'essenza della città e dei suoi abitanti. La sua non è mera registrazione della vita, le immagini che lei carpisce non sono spunti per dei quadretti di costume (che tuttavia non mancano); tendono invece a cristallizzarsi in simboli del nostro tempo veicolando le riflessioni metafisiche dell'autrice:

Il percorso che faccio più spesso dal Gianicolo al centro della città è la strada che attraverso Trastevere, piazza Argentina e piazza Venezia porta ai Fori Imperiali, dalla Colonna Traiana fino al Colosseo. Quello che mi trascina in questi luoghi non è tanto l'amore per l'antichità quanto il piacere che mi dà la vita tra le rovine. I pigri che si crogiolano al sole sui gradini del Campidoglio, la bella Aleksandra di Cracovia che dà da mangiare 
ai gatti ai piedi della rupe Tarpea, il corteggiamento dei cani nel Foro di Cesare. Amo osservare i bambini che costruiscono le proprie basiliche di sabbia, dove le macerie dei templi e dei mercati ridotte in polvere si mescolano a quella sollevata dalle limousine dei ministri democristiani (129).

Con sottile ironia, l'autrice prende le distanze dal tradizionale viaggio romano di romantica memoria: Klasyczna promenada ("La passeggiata classica") - questo è il titolo del saggio in questione - non è animata dall'interesse per l'antico ma dal piacere di osservare il contrasto tra le rovine e lo scorrere incessante della vita. Senza dubbio il trasformarsi delle rovine in polvere prefigura un'opposizione natura-cultura, passato-presente, che è di chiara ascendenza romantica. I riferimenti al romanticismo in questo saggio sono espliciti: l'immagine di un'antica casa abbandonata, rovina sopraffatta e invasa dalla natura, le fa venire in mente il passato e i giardini di Stendhal: "Forse era qui che veniva durante le sue Passeggiate romane?” (83), si domanda l'autrice. Tuttavia ad animarla non è la ricerca delle tracce romane dei romantici (come faceva per esempio Iwaszkiewicz): il punto di arrivo della sua riflessione è ancora una volta la trascendenza e il bisogno di eternità. La natura del parco di Villa Sciarra è "senza peccato", espressione di "un mondo non toccato dalla curiosità di Adamo ed Eva" e la scrittrice si ferma davanti a una magnolia "come davanti a un altare" (87). Ma la natura, i platani coperti di gemme, non impediscono al suo sguardo di abbracciare la città:

Ora la luce su di lei [Roma] è dorata. Tuttavia non è la pallida doratura del limone, ma quella scura del miele. Ed è così densa che sembra spalmata sugli stretti mattoni, sulle pietre cristiane e pagane. Eguaglia i secoli, eguaglia le fedi, fa di tutto questo un solo blocco prezioso. [...] Lungo il fiume sfilano silenziose scatolette di tutti i colori. Si odono i rintocchi di una campana. Uno, due, dieci, tutti smorzati. Vedo la città dove abitano il tempo, il silenzio e la voce delle chiese. L'immateriale materialità. Lo scorrere senza movimento. La staticità della storia (87-88). 
L'immagine della Roma di Kuncewiczowa nasce sul varco e nello scontro tra queste due traiettorie poetiche, speculative, metafisiche: quella orizzontale, volta al presente, all'immanenza, alla materia - quella verticale, orientata all'eterno, alla trascendenza, allo spirito. Liberarsi dalla prigionia angustiante del tempo e della materia, perdersi nella contemplazione di Roma o nella folla, cercare di ricondurre a un senso od ordine superiore il caos della metropoli contemporanea: è questa l'urgenza che si avverte nelle pagine di Notatki wtoskie, un libro che si radica nel presente trascendendolo.

La scrittrice osserva stupita sullo sfondo del paesaggio romano, con lo sguardo di una straniera, i tanti volti della civiltà urbana contemporanea, in un'epoca in cui - nonostante la cortina di ferro il mondo già si avviava a quella mescolanza e contaminazione transculturale, alla folla carnevalesca che anima oggi le vie delle grandi metropoli del mondo. Non al passato e ai suoi segni è dunque rivolto il suo sguardo, ma al divenire incessante del presente. Si osservi il seguente frammento, dove la realtà della strada è ricostruita con un efficientissimo accumulo, testimonianza di una scrittura istintiva, emotiva, imprevedibile:

Bianche bambinaie a spasso con neri neonati, menestrelli americani in calzoni rattoppati che cantano la fratellanza dei popoli e delle classi sociali, geishe in jeans scortate da samurai in pantaloncini corti, gitane senza il fiore di camelia dietro l'orecchio accompagnate da caballeros senza pistole dietro la cintura, testimoni di Geova che annunciano con zelante discrezione la fine del mondo, studenti di mille università, artisti di arti non riconosciute, apostoli di fedi non scritte, vittime del capitalismo, vittime del comunismo, trionfatori di olimpiadi non disputate, professori di sociologia col revolver dalle pallottole avvelenate, tossicodipendenti della Grande Illusione, Aspasie, Antigoni e Cleopatre della quotidianità... Mi piace passare di là. Seguire lo zigzagare dei ricordi, i sentieri dei sogni, le strade impervie di quello che è stato, e non è più. Mi piace camminare come il pendolo di un orologio, senza pensare al tempo. Adoro i Fori Imperiali non per i loro cesari, 
ma per la persistenza della pietra, dei gatti, dei cani, dei neonati e degli asfodeli. I cattivi imperi e i buoni imperi sono trascorsi, ma non è trascorso il mondo (130).

A Roma rifluisce l'umanità, nei suoi infiniti travestimenti, nelle sue continue combinazioni, ideologiche, etniche, culturali. Roma è una città piena di ambivalenze, contraddizioni perché - come scrive Kuncewiczowa - ha "tanti cassetti, da cui si riversano epoche, aneddoti, biografie, fatti e antefatti, persone reali e fantastiche. Estraggo a casaccio paesaggi, allucinazioni, ritratti, spettri, vestiti di donne che non ci sono più e i miei travestimenti” (83). Eppure resta qualcosa di eterno e immutabile in Roma, anche se "la città dove abita il tempo", non può essere colta e compresa nella sua essenza. Perché il mistero che racchiude è il mistero dell'uomo e della morte. Roma in fondo non è che "un grande sepolcreto".

Finché è arrivata quella sera in cui Roma mi è sembrata totalmente consumata. Gli antichi fori che terminano con quella colossale rotonda dove tante volte gli animali sono stati aizzati contro gli uomini e gli uomini contro gli animali apparivano ingrigiti, come coperti di milioni di parole etrusche, gotiche, galiziane, giapponesi, polacche e chissà ancora di chi. Quelle parole giacciono in uno spesso strato sulle piazze dimenticate, sui templi mutilati, su tutta quella maestosità incomprensibile e indescrivibile. Con irritazione ho fermato i miei pensieri sulla soglia delle cucine dove non si cucina, delle piazze del mercato senza merci, dei palazzi dove non si abita, dei templi senza dei ignoti: un grande sepolcreto (117-118).

\section{Conclusioni}

La sua particolare condizione di sradicata e di "straniera" offriva a Maria Kuncewiczowa uno sguardo disincantato sulle cose, una prospettiva obliqua che le permetteva di cogliere i sensi nascosti sotto la superficie della realtà. È questa una caratteristica che, fa notare Krystyna Jaworska, la scrittrice condivide con le eroine dei 
sui romanzi ${ }^{12}$. Notatki wtoskie si collocano nel filone di quelle opere polacche novecentesche dove il viaggio in Italia, e a Roma in particolare, non può prescindere da due funzioni precipue: la ricerca di ispirazione artistica e di soddisfazione intellettuale, da un lato, la volontà di offrire un giudizio personale e inedito sulla crisi del mondo contemporaneo. Tutto questo è già incluso nella tradizione polacca del genere: già Norwid nel poema Szczesna aveva accostato la grande magnificenza delle rovine di Paestum con la descrizione delle miserabili condizioni di vita della popolazione. Ma Roma e l'Italia per Kuncewiczowa non hanno alcuna funzione consolatoria, l'autrice non cerca rifugio in una seconda patria ideale o nel mito, non è alla ricerca di modelli di eterna bellezza, come fanno gli epigoni contemporanei dei romantici (Iwaszkiewicz in primis). Giunta alla fine della sua vita, l'ispirazione e la pienezza agognate dalla Kuncewiczowa sono prima di tutto di natura spirituale.

Anche i richiami e i contrappunti polacco-italiani disseminati nel libro devono essere colti nel contesto della migliore tradizione romantica; si va da suggestioni intime e private a echi artistici, letterari e culturali: due mamme romane che giocano a rincorrersi con i loro bambini le ricordano la sua infanzia all'inizio del secolo, al parco di Ujazdów (88); il soffiare dello scirocco e le vie laterali del Gianicolo, con i giardinetti ombreggiati con le foglie di palma, le fanno tornare alla memoria un affresco semicancellato della cappella della Santa Trinità a Lublino. E rientra in questo filone tradizionale anche il complesso polacco, l'idea che la Polonia non sia adeguatamente compresa, la sensazione di essere percepiti come "barbari nel giardino": tutti aspetti riscontrabili anche in altre opere del viaggio in Italia dell'epoca [cfr. Prola 2018: 24-37]. La scrittrice constata che nella mostra dei Musei Vaticani dedicata

Scrive Jaworska [2016: 162]: "molti dei suoi personaggi [di Kuncewiczowa] sono donne che, in quanto provenienti da un altrove, vivono sapendo di essere percepite come aliene e la mancata identificazione è per loro fonte di disagio. Oscillano tra estraneità e familiarità. Sentono la propria alterità come una condanna e un privilegio, in quanto essere in qualche modo estranee permette loro di osservare la società in cui vivono in una duplice prospettiva, interna ed esterna al tempo stesso, e di rapportarsi a essa con ambivalenti sentimenti di appartenenza e disappartenenza". 
alla storia delle religioni, non vi è un solo oggetto riguardante il culto degli antichi slavi; sulla mappa dei beni importati dagli antichi Romani "figurano pellicce, ambre, ceramiche che sembrano venire da Stara baśń" (96). Il riferimento al primo romanzo del ciclo Dzieje Polski di Józef Ignacy Kraszewski, vuole sottolineare che anche da quella parte d'Europa dove si trova la Polonia, oltre il limes Imperii, proveniva quel fasto che adornava le case dei romani. E questo è bene ricordarlo, perché la consapevolezza e l’orgoglio delle proprie origini, la percezione del valore apportato da ogni cultura, resta indispensabile anche in un'epoca in cui "nessuno può più dirsi a casa sua”.

Quello di Kuncewiczowa è uno sguardo incantato e disincantato a un tempo, distrattamente attento alla realtà, contemplante, saggio e candido a un tempo. Distratto, nel senso etimologico di distrahĕre, dunque tolto, sottratto da un luogo, vagolante in un altrove dove i poeti trovano nutrimento per la propria arte, al di sotto delle apparenze, oltre la materialità del mondo; contemplante perché la scrittrice non sfugge dalla realtà in un mondo di paradisiaca e consolatoria illusione, culturale o fideistica, bensì "raccoglie nel proprio orizzonte", nel proprio templum, e instancabilmente interpreta il senso di ciò che intravede.

\section{Bibliografia}

Barycz Henryk (1965), Rola Rzymu w kulturze polskiego oświecenia, in: Id., Spojrzenia w przeszłość polsko-włoska, Zakład Narodowy im. Ossolińskich, Wrocław.

Biliński Bronisław (1992), Figure e momenti polacchi a Roma. Strenna di commiato, Zakład Narodowy im. Ossolińskich, Wrocław.

Czapliński Przemysław (1998), Ślady przełomu, o prozie polskiej 1976-1996, Wydawnictwo Literackie, Kraków.

Glensk Urszula (2014), Historia słabych. Reportaż i życie w Dwudziestoleciu (1918-1939), Universitas, Kraków.

Gutkowska Barbara (1988), Poetyka i problematyka „Przezroczy” Marii Kuncewiczowej, in: W stronę Koncewiczowej. Studia i szkice, a cura di Włodzimierz Wójcik, Uniwersytet Śląski, Katowice, pp. 59-75. Jaworska Krystyna (2016), La straniera di Maria Kuncewiczowa e altre madri aliene, in: Disappartenenze. Figure del distacco e altre solitudini 
nelle letterature dell'Europa centro-orientale, a cura di Ljiljana Banjanin, Krystyna Jaworska, Massimo Maurizio, Stilo Editrice, Bari, pp. 149-173.

Kąkolewski Krzysztof (1992), Reportaż, in: Stownik literatury polskiej XX wieku, a cura di Alina Brodzka et al., Zakład Narodowy im. Ossolińskich, Wrocław, pp. 930-935.

Kozińska-Donderi Diana (2003), Obraz Włoch i motywy włoskie w prozie polskiej 1918-1956, Wydział Filologiczny Uniwersytetu Wrocławskiego, Wrocław.

Kuncewiczowa Maria (1935), Il volto dell'uomo, trad. di Silvana Lupo, Cafaro, Roma-Lecce.

Kuncewiczowa Maria (1939), La straniera, trad. di Renato Poggioli, Mondadori Milano.

Kuncewiczowa Maria (1971), Fantomy, Pax, Warszawa.

Kuncewiczowa Maria (2010), Notatki włoskie. Przezrocza, Feeria, Łódź. Piechota Magdalena, Stępnik Krzysztof (2004) (a cura di), Reportaż $w$ dwudziestoleciu międzywojennym, Wydawnictwo UMCs, Lublin.

Prola Dario (2018), „Spossato dalla bellezza”. L'Italia nella scrittura di Jaroslaw Iwaszkiewicz, Edizioni dell'Orso, Alessandria.

Statuti Paolo (1988) (a cura di), Viaggio sulla cima della notte. Racconti polacchi dal 1945 ad oggi, Editori Riuniti, Roma.

Świętosławska Teresa (1997), Ostatni „dylizans” Marii Kuncewiczowej, in: O twórczości Marii Kuncewiczowej, a cura di Lech Ludorowski, Wyd. Uniwersytetu Marii Curie-Skłodowskiej, Lublin, pp. 97-105.

Szłagan Alicja (2010), Maria Kuncewiczowa w podróży, in: Ead., Maria Kuncewiczowa - przyblizenia. Szkice biograficzne, Wydawnictwo IBL, Warszawa, pp. 196-216.

Wilkoń Teresa (2006), Nimfy oko btękitne: obrazy Neapolu w poezji polskiej XIX i XX wieku, Wydawnictwo Uniwersytetu Śląskiego, Katowice.

Zaworska Helena (1983) (a cura di), Rozmowy z Marią Kuncewiczowa, Czytelnik, Warszawa.

Dario Prola

"Where the time, silence and the voice of the churches live": the Rome of Maria Kuncewiczowa

This paper is focused on a volume of short essays and notes by Maria Kuncewiczowa entitled Notatki wtoskie. Przezrocza (Italian notes. Slides) from 1985. The first part is devoted to the evolution and the literary realizations of the Italian journey, starting from 1918, in order to offer an interpretation of the 
place occupied by Kuncewiczowa's work in the panorama of contemporary Polish literature. In the second part, the author analyzes the genesis and genre of this literary text, recognizing its characteristics as a silva rerum, a traditional formal model of Polish literature which experienced renewed fortune in the second half of the twentieth century. The third part of the essay constitutes its thematic core: the author analyzes the image of Rome in all its many facets, relating it to the writer's poetics and worldview.

Keywords: Kuncewiczowa; Italian journey; Roma; Aldo Moro; Karol Wojtyła.

Dario Prola - lavora presso l'Istituto di Comunicazione Specialistica e Interculturale, Facoltà di Linguistica Applicata dell'Università di Varsavia dove dirige la Sezione italiana. Insegna Storia della letteratura italiana e tiene corsi sulla traduzione letteraria e i linguaggi specialistici. Si occupa di letteratura contemporanea italiana e polacca, letteratura comparata, traduzione letteraria. Ha pubblicato numerosi articoli scientifici su scrittori italiani e polacchi. Tra i suoi lavori si menzionano i volumi Mito e rappresentazione della città nella letteratura polacca (Aracne, 2014); "Spossato dalla bellezza". L'Italia nella scrittura di Jarostaw Iwaszkiewicz (Edizioni dell'Orso, 2018); Il traduttore errante (con E. Jamrozik, Uniwersytet Warszawski, 2017); Sponde, confini, trincee: l'Italia nell'Europa post-1918 (con S. Rosatti, DiG 2019); Rok 1968 $w$ Europie Badania i pamięć / The Year 1968 in Europe Research and Memory (con P. Podemski, Scholar, 2019). È membro del comitato scientifico della collana «Italipolis» (DiG) e del comitato redazionale delle riviste «PL.IT / Rassegna italiana di argomenti polacchi $\gg$ e $\ll$ Kwartalnik Neofilologiczny». Ha tradotto in italiano diversi autori polacchi del Novecento. 
